\title{
The magnetic field effect on the transport and efficiency of group III tris(8-hydroxyquinoline) organic light emitting diodes
}

\author{
P. Shakya, P. Desai, M. Somerton, G. Gannaway, T. Kreouzis, and W. P. Gillin ${ }^{\text {a) }}$ \\ Department of Physics, Queen Mary, University of London, Mile End Road, London E1 4NS, \\ United Kingdom
}

(Received 14 December 2007; accepted 23 March 2008; published online 28 May 2008)

\begin{abstract}
Magnetoresistance and efficiency measurements of organic light emitting diode structures based on the group III hydroxyquinolates $\left(M q_{3}\right)$ have been made as a function of magnetic field and $M q_{3}$ thickness, where $M=\mathrm{Al}, \mathrm{Ga}$, and In. For all quinolates, independent of thickness, we observed very similar behavior for the efficiency of the devices, with large increases in efficiency occurring at low values of applied field, which rapidly saturate as the field is increased. The current through these devices is found to be a strong function of both the device thickness and the metal ion. For $\mathrm{Al}_{3}$ based devices, the current changes appear to correlate strongly with the triplet population in the devices. For $\mathrm{Ga}_{3}$ and $\mathrm{In} q_{3}$ devices, the magnetoresistance is found not to correlate with the triplet concentration and this may be evidence that there is little energetic barrier for carrier trapping in these materials. For all materials, a further dependence of the magnetoresistance on applied field was observed, which needs closer investigation. () 2008 American Institute of Physics.
\end{abstract}

[DOI: $10.1063 / 1.2932079]$

\section{INTRODUCTION}

The effect of magnetic fields on the light output and current in organic light emitting diodes (OLEDs) has been receiving increased research interest since the first observation in $2003^{1}$ that a magnetic field significantly increased both the light emission and the current through such a device. Mermer et al. ${ }^{2-4}$ showed that the effect of the magnetic field on the current through organic devices was a general phenomenon and could be observed in a range of both the small molecule and the polymer systems. They dubbed the effect of organic magnetoresistance (OMR). Since then, there has been relatively little quantitative work into the detailed mechanisms behind the phenomenon although the role of both the hyperfine interactions and the spin-orbit effects has been investigated. ${ }^{5,6}$ Despite the lack of quantitative work on the mechanisms behind OMR, there are several possible mechanisms that have been proposed. Prigodin et al. ${ }^{7}$ suggested that the effect of the magnetic field is to change the ratio between the singlet and the triplet generation that has the effect of increasing the recombination rate and hence increase the current. Recently, Bobbert et al. ${ }^{8}$ proposed a mechanism that is based on the hopping of polarons and bipolaron formation and Desai et al. ${ }^{9}$ suggested that the magnetic field can change the intersystem crossing between the singlet and the triplet state, and the corresponding effect on triplet populations would change the trapping rate of carriers on these excited states.

This model was based on the work performed in the 1960s and 1970s where the role of magnetic fields on the luminescence of organic molecules and, in particular, on the transfer between the singlet and triplet states was widely studied. The main effects that operate to alter the singlettriplet ratio are magnetic hyperfine interactions, which act to

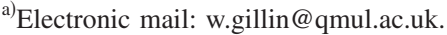

allow interconversion between the singlet and triplet, $T_{0}$, states $^{10,11}$ and the magnetic field effect (MFE) on the quenching of triplets through triplet-triplet interactions. ${ }^{12-14}$ The role of magnetic fields on the interaction between triplets and paramagnetic centers, such as free carriers, was studied in anthracene crystals by Ern and Merrifield. ${ }^{15}$ They showed that the quenching of a triplet exciton by a paramagnetic center (such as free charge carriers) would be suppressed by the presence of a magnetic field and that this can be seen as an increase in the triplet lifetime.

We have recently studied the effect of changing the thickness of the $\mathrm{Al} q_{3}$ layer in an OLED on the current transport. ${ }^{16} \mathrm{We}$ found that the thickness of the layer played a major role in the OMR observed with thin devices showing a negative OMR at low drive current, which became positive as both the drive current and the applied magnetic field were increased. For thicker devices, the OMR was found to always be positive. By comparison with the observed changes in efficiency that were measured in these devices, a qualitative model for the OMR was proposed. This model was based, in part, on the interaction between the charge carriers in the device and triplet excitons. For thin devices, the magnetic field induced reduction in triplet concentrations had an additional effect in that it reduced the magnitude of carrier recycling caused by triplets dissociating at the cathode and this resulted, at low current density, in a reduction in the current through the device. ${ }^{16,17}$ In addition, the lower triplet population caused a reduction in the degree of interaction between the triplets and the charge carriers, which increased the mobility and caused an increase in the current through the device. The overall effect on the current was a combination of these two processes.

In this paper, we have extended the work to investigate the role of changing the central ion in the quinolate layer from aluminum to gallium and indium. We have found that 
this change has little effect on the efficiency of the devices but that it dramatically reduced the magnitude of the OMR. This shows that it is possible to get identical increases in efficiency in all the quinolates, while for $\operatorname{In} q_{3}$, this increase in efficiency is not accompanied, at least at low fields, by any change in the current through the device.

\section{EXPERIMENTAL METHOD}

The basic device structure consists of an indium tin oxide (ITO coated glass substrate (purchased from Merck) with a sheet resistivity of $\sim 13 \Omega / \square, \quad 500 \AA$ of $N, N^{\prime}$-diphenyl- $N, N^{\prime} \quad$ bis(3-methylphenyl)-(1, $1^{\prime}$ -biphenyl)-4, $4^{\prime}$ diamine (TPD) as the hole transport layer. For the emissive layer/electron transport layer between 100 and $900 \AA$, of either $\mathrm{Al} q_{3}, \mathrm{Ga} q_{3}$, or In $q_{3}$ was deposited. On to these devices, a cathode was deposited consisting of a $10 \AA \mathrm{LiF}$ layer followed by $1000 \AA$ of aluminum. The TPD and $\mathrm{Al}_{3}$ were purchased from Aldrich while the $\mathrm{Ga}_{3}$ and In $q_{3}$ were synthesized according to literature (Ref. 18 and references therein); all materials were purified by train sublimation prior to use. The ITO substrate was patterned using photolithography and cleaned by ultrasonicating in detergent solution, water, acetone, and chloroform. Following this, the ITO was treated in an oxygen plasma for $3 \mathrm{~min}$ at $30 \mathrm{~W}$ and 2.5 mbar pressure by using a Diener Electronic Femto Plasma system. The plasma treated substrate was immediately transferred to the deposition chamber for device fabrication. The deposition of the organic layers and metal electrodes was performed by using a Kurt J. Lesker SPECTROS evaporation system with a base pressure during evaporation of $\sim 10^{-7}$ mbar. The rate of deposition of organic materials was about $2 \AA / s$ while that of the aluminum was varied from $\sim 1$ to $10 \AA / \mathrm{s}$. A calibrated oscillating quartz crystal monitor was used to determine the rate and thickness of the deposited layer. The whole device fabrication was performed without breaking vacuum.

Immediately after growth, the devices were placed in a light-tight sample holder with a calibrated silicon photodetector (Newport 818-SL) placed on the top surface of the device. The sample holder was placed between the poles of an electromagnet with the magnetic field perpendicular to the direction of current flow in the device. The photodetector was tested under various illumination levels to make sure that there was no field dependence on its output. Measurements were taken with the device operated in constant voltage mode. Before and after each field measurement, a measurement at null field was taken. These two readings were averaged and used to determine the effect of the magnetic field. This procedure was performed in order to remove any effects due to drifting in the device characteristics. Voltage sourcing and current measurements were performed by using a Keithley 236 source-measure unit with current measurements being averaged over 32 readings. The optical power output was measured by using a Newport 1830 optical power meter.

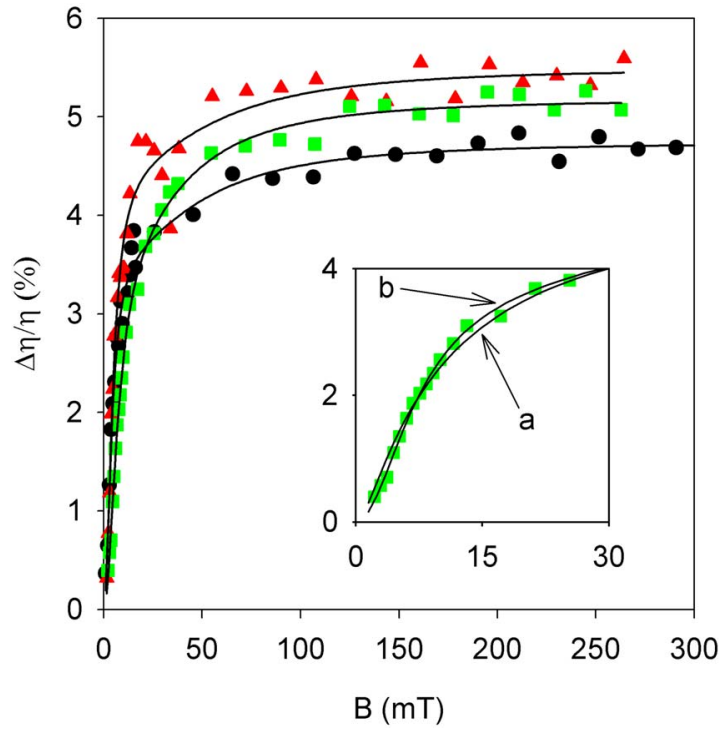

FIG. 1. (Color online) The change in device efficiency for $90 \mathrm{~nm}$ devices at just after device turn-on. Circles-Al $q_{3}$, triangles- $\mathrm{Ga} q_{3}$, and squares-In $q_{3}$. The inset shows the In $q_{3}$ data at low field along with the (a) $B^{2} /\left(|B|+B_{0}\right)^{2}$ fit and the (b) $A_{0} B^{2} /\left(B^{2}+B_{0}^{2}\right)+A_{1} B^{2} /\left(B^{2}+B_{1}^{2}\right)$ fit.

\section{RESULTS AND DISCUSSION}

Figure 1 shows the change in efficiency with applied field for devices with $90 \mathrm{~nm}$ of either $\mathrm{Al} q_{3}, \mathrm{Ga} q_{3}$, or In $q_{3}$. In each case, it can be seen that the increase in efficiency is roughly comparable for the three devices. This was seen for all device thicknesses and current densities within the device. In our earlier work, we suggested that this increase in efficiency was due to a magnetic field dependence in the intersystem crossing rate between the singlet and triplet states. The fact that these changes in efficiency are so similar for the three materials, for a given current density, suggests that the $B$ dependence of the intersystem crossing is not greatly perturbed by the difference in the central ion in the quinolate layer. Whether the absolute value of the null field intersystem crossing changes cannot be determined from these measurements.

Close inspection of the curves in Fig. 1 shows that although the magnitude of the change in the intersystem crossing at high field is relatively independent of the central ion mass, the shape of the curves is subtly different. Mermer et $a l .{ }^{4}$ suggested the observation that the OMR in various organic materials can be fitted empirically with an equation of the form $B^{2} /\left(B^{2}+B_{0}^{2}\right)$ or $B^{2} /\left(|B|+B_{0}\right)^{2}$, the first of which they describe as a "Lorenzian." They went on to show that the first of these expressions can be related to the effect of the hyperfine interaction. ${ }^{5}$ In a later paper, they went on to suggest that the fact that some of their OMR data appeared to have two processes, which could be fitted with a pair of these expressions, could be evidenced that both the hyperfine and the spin-orbit effects were visible, which depend on the material system. ${ }^{6}$ They show how the first of these expressions can be deduced from both the hyperfine and the spin-orbit Hamiltonians but state that they are not yet able to deduce the second function. We have tried to fit our efficiency data by using each of these expressions and found that the second expression gives a much better fit to the data. However, close 
TABLE I. The fitting parameters obtained from the efficiency data as a function of applied field for all of the $\mathrm{Al} q_{3}$, Ga $q_{3}$, and In $q_{3}$ devices under two drive conditions.

\begin{tabular}{|c|c|c|c|c|c|c|c|c|c|c|c|c|c|}
\hline & \multirow[b]{2}{*}{$J\left(\mathrm{~A} / \mathrm{m}^{2}\right)$} & \multicolumn{4}{|c|}{$\mathrm{Al} q_{3}$} & \multicolumn{4}{|c|}{$\mathrm{Ga} q_{3}$} & \multicolumn{4}{|c|}{$\operatorname{In} q_{3}$} \\
\hline & & $A_{0}$ & $B_{0}(\mathrm{mT})$ & $A_{1}$ & $B_{1}(\mathrm{mT})$ & $A_{0}$ & $B_{0}(\mathrm{mT})$ & $A_{1}$ & $B_{1}(\mathrm{mT})$ & $A_{0}$ & $B_{0}(\mathrm{mT})$ & $A_{1}$ & $B_{1}(\mathrm{mT})$ \\
\hline \multirow[t]{2}{*}{$90 \mathrm{~nm}$} & 0.1 & 3.7 & 4.1 & 1.1 & 52 & 4.5 & 4.8 & 1 & 60 & 3.8 & 7.3 & 1.4 & 44 \\
\hline & 100 & 1.5 & 4.6 & 0.9 & 80 & 2.1 & 5.3 & 1.2 & 112 & 2.1 & 9.0 & 0.8 & 121 \\
\hline \multirow[t]{2}{*}{$50 \mathrm{~nm}$} & 0.1 & 4.4 & 2.7 & 2.7 & 17 & 4.7 & 4.2 & 1.5 & 30 & 3.9 & 7.1 & 1.0 & 44 \\
\hline & 100 & 2.4 & 3.6 & 1.0 & 53 & 2.2 & 4.8 & 1.1 & 81 & 1.5 & 6.7 & 0.8 & 19 \\
\hline \multirow[t]{2}{*}{$30 \mathrm{~nm}$} & 0.1 & 4.1 & 2.6 & 3.4 & 11.8 & 4.4 & 4.0 & 1.2 & 26 & 4.7 & 7.5 & 1.3 & 54 \\
\hline & 100 & 3.0 & 3.3 & 1.2 & 27.5 & 2.2 & 4.4 & 0.8 & 60 & 2.2 & 8.3 & 0.6 & 68 \\
\hline \multirow[t]{2}{*}{$20 \mathrm{~nm}$} & 0.1 & 5.3 & 2.8 & 3.4 & 14 & 4.7 & 4.2 & 1.3 & 23 & 5.1 & 7.4 & 1.7 & 51 \\
\hline & 100 & 2.7 & 3.2 & 1.0 & 32 & 2.3 & 4.6 & 1.0 & 59 & 3.0 & 8.2 & 0.5 & 87 \\
\hline \multirow[t]{2}{*}{$15 \mathrm{~nm}$} & 0.1 & 5.9 & 2.7 & 3.5 & 16 & 5.2 & 4.2 & 1.2 & 37 & 5.2 & 7.7 & 1.6 & 58 \\
\hline & 100 & 3.1 & 2.9 & 1.5 & 16 & 2.6 & 4.2 & 0.8 & 30 & 2.8 & 8.2 & 0.6 & 57 \\
\hline
\end{tabular}

inspection shows that at low fields, the fit is poor and we have therefore tried to fit the data by using an expression of the form $A_{0} B^{2} /\left(B^{2}+B_{0}^{2}\right)+A_{1} B^{2} /\left(B^{2}+B_{1}^{2}\right)$, which is what might be expected if there were both a hyperfine and a spinorbit interaction affecting the intersystem crossing from the triplet to the singlet state (inset in Fig. 1). This gives an excellent fit to all our efficiency data for each material at all magnetic fields and current densities. Table I gives the resulting fitting parameters for each quinolate at different device thicknesses at just after device turn-on $\left(\sim 0.1 \mathrm{~A} / \mathrm{m}^{2}\right)$ and under typical operating conditions of $\sim 100 \mathrm{~A} / \mathrm{m}^{2}$. The values for the low field process are reasonably well defined for each curve, which results in small variations in the $B_{0}$ terms for each material. For the high field process, the fits are less well defined, which leads to greater variations in the $B_{1}$ values. However, if we take the average value of the critical field, from all the data, for each material, we obtain values for $B_{0}$ of $3.25,4.5$, and $7.7 \mathrm{mT}$ and for $B_{1}$ of 36,52 , and 60 $\mathrm{mT}$ for $\mathrm{Al} q_{3}, \mathrm{Ga} q_{3}$, and $\mathrm{In} q_{3}$ respectively. As noted by Nguyen et al., the lower field value would be expected to relate to the hyperfine interaction, whereas the higher field component would be expected to be due to the spin-orbit interaction. However, it is not at all clear why changing the mass of the central ion in these complexes should have an effect on the hyperfine interaction, which should be dominated by the hydrogen atoms nearest to the exciton.

Figure 2 shows the OMR data for the $90 \mathrm{~nm}$ devices, operated at a current density of $\sim 100 \mathrm{~A} / \mathrm{m}^{2}$. The $\mathrm{Al} q_{3}$ data show a clear similarity with the efficiency data as a function of magnetic field, namely, an initial rapid rise in current at small values of $B$. However, at higher applied fields rather than saturate, as the efficiency does, the OMR continues to increase with increasing field. For the $\mathrm{Ga} q_{3}$ and $\operatorname{In} q_{3}$ samples, the measured OMR is very different. Here, there is no rapid rise in the OMR at low field, unlike the efficiency curves for these devices, but the OMR appears to increase approximately linearly with increasing $B$. The solid line fit in Fig. 2 for the $\mathrm{Al}_{3}$ data was obtained by using the dual "Lorentzian," used to fit the efficiency, with an additional linear term. The values of the critical field obtained for the two Lorentzians in this fit are 6.4 and $67 \mathrm{mT}$, which are comparable to the values obtained from the efficiency data for this sample. This result for $\mathrm{Al}_{3}$ suggests that if the mo- bility of the carriers is affected by the presence of triplets within the device, then there is also a $B$ dependence of this interaction, which increases with increasing $B$ field. For the $\mathrm{Ga} q_{3}$ and $\mathrm{In} q_{3}$ samples, the $B$ dependence of the OMR appears to be approximately linear and does not show the Lorentzian components, which are present in the efficiency data for these samples. This implies that the interaction between the carriers and triplets at null field is weak for these materials, which suggests that for $\mathrm{Ga} q_{3}$ and $\operatorname{In} q_{3}$ at null field, there is little energetic barrier for a carrier trapped at a triplet site, and so the increase in the triplet concentration has a negligible effect of the mobility at low magnetic fields. In addition, the observation that, for example, at a field of 30 $\mathrm{mT}$, there is effectively no increase in the current through the device while there is a $1.5 \%-3 \%$ increase in the efficiency of the device is strong evidence that the change in efficiency cannot be a consequence of the OMR. This observation cannot be simply explained within the Prigodin model. ${ }^{7}$

Although in Fig. 2 we have used a linear function to fit the field dependence of the OMR, this is an approximation with no physical significance and is used solely to highlight

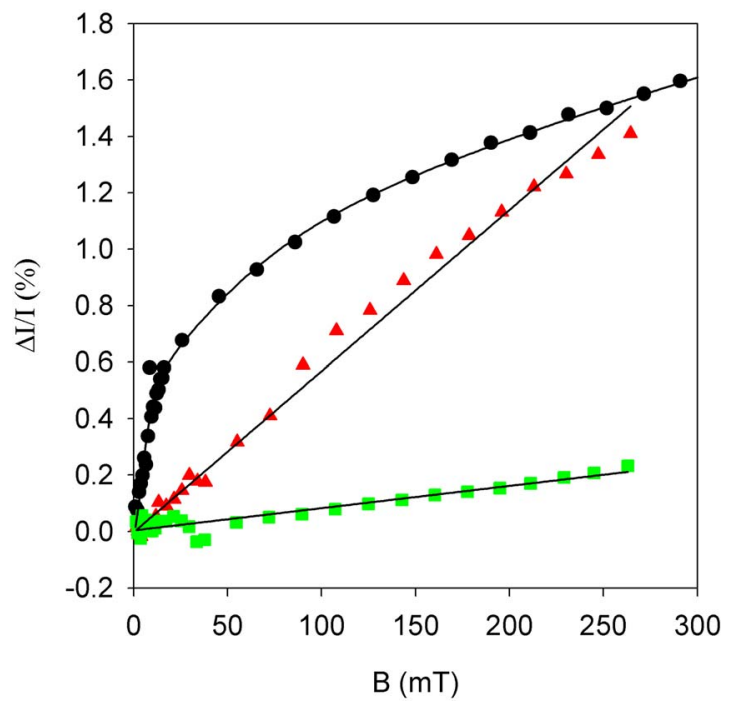

FIG. 2. (Color online) The percentage change in current through the $90 \mathrm{~nm}$ devices at a current density of $\sim 100 \mathrm{~A} / \mathrm{m}^{2}$. Circles- $\mathrm{Al} q_{3}$, triangles- $\mathrm{Ga} q_{3}$, and squares- $\operatorname{In} q_{3}$. 


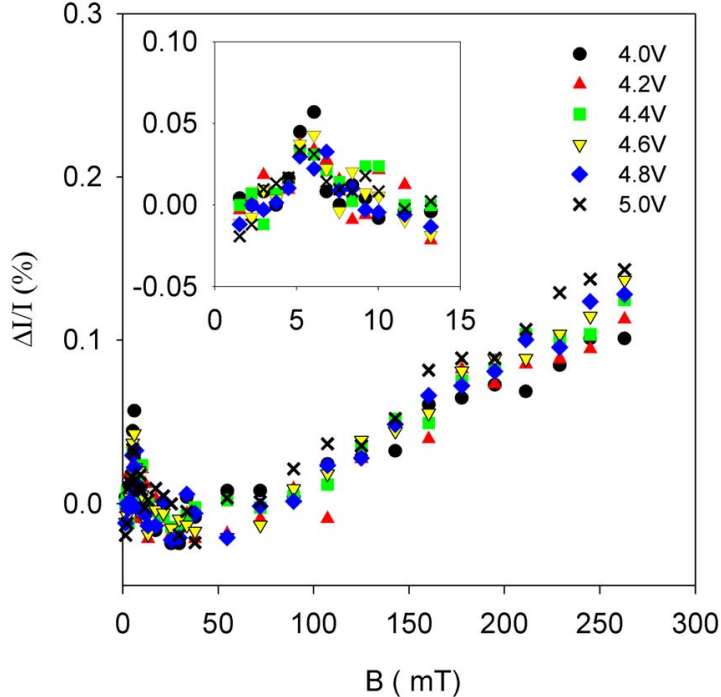

FIG. 3. (Color online) The percentage change in current through the $90 \mathrm{~nm}$ In $q_{3}$ device at drive voltages from 4.0 to $5.0 \mathrm{~V}$. The inset shows the data at low fields.

the differences in the OMR for the three materials. Closer analysis of the $\operatorname{In} q_{3}$ samples has shown that there are more complicated effects, which occur at low magnetic fields. In Fig. 3, we present data from the $900 \AA \operatorname{In} q_{3}$ device at drive voltages from 4 to $5 \mathrm{~V}$; the inset shows the region below 15 mT. From Fig. 3, it can be seen that there is a small peak in the OMR that occurs at $\sim 6 \mathrm{mT}$. This peak is small, with a maximum value of $<0.05 \%$, and is only just discernible from the noise in the data. However, as can be seen in Fig. 3, it is a consistent feature at all drive voltages. Following this small peak, the data go slightly negative before rising from fields of $\sim 50 \mathrm{mT}$. This feature is not visible in the $900 \AA$ $\mathrm{Ga} q_{3}$ or $\mathrm{Al}_{3}$ data.

In Fig. 4(a), the low field peak can clearly be seen for the $200 \AA \mathrm{Ga} q_{3}$ device. For other thicknesses, it is present but is visible only as a shoulder on the rising background of the OMR. Figure 4 shows the low field OMR for the 200 and $500 \AA \mathrm{Ga} q_{3}$ devices at different current densities. The $500 \AA \mathrm{Ga} q_{3}$ device [Fig. 4(b)] also exhibits another effect, which is also seen in the thinner $\operatorname{In} q_{3}$ devices; as the current density through the device is increased, the magnitude of this peak reduces before becoming negative. It can also be seen from Fig. 4(b) that as the current density in the device increases and the peak at $\sim 6 \mathrm{mT}$ becomes negative, the first data point at $1.7 \mathrm{mT}$ increases in intensity. This increase at very low fields appears to be very reproducible and is seen in a number of samples, and presumably is evidence of a peak at even lower magnetic fields. However, with our current experimental setup, we are unable to reliably obtain fields smaller than $\sim 1-2 \mathrm{mT}$ in order to accurately investigate this phenomenon. At the present time, it is unclear what is responsible for these processes. It may be that they are related to the $B$ dependence of the interaction between a carrier and a triplet or they could be due to some other phenomena such as the bipolaron mechanism recently suggested by Bobbert $e t a l{ }^{8}$ Further work will be needed to investigate these interactions.
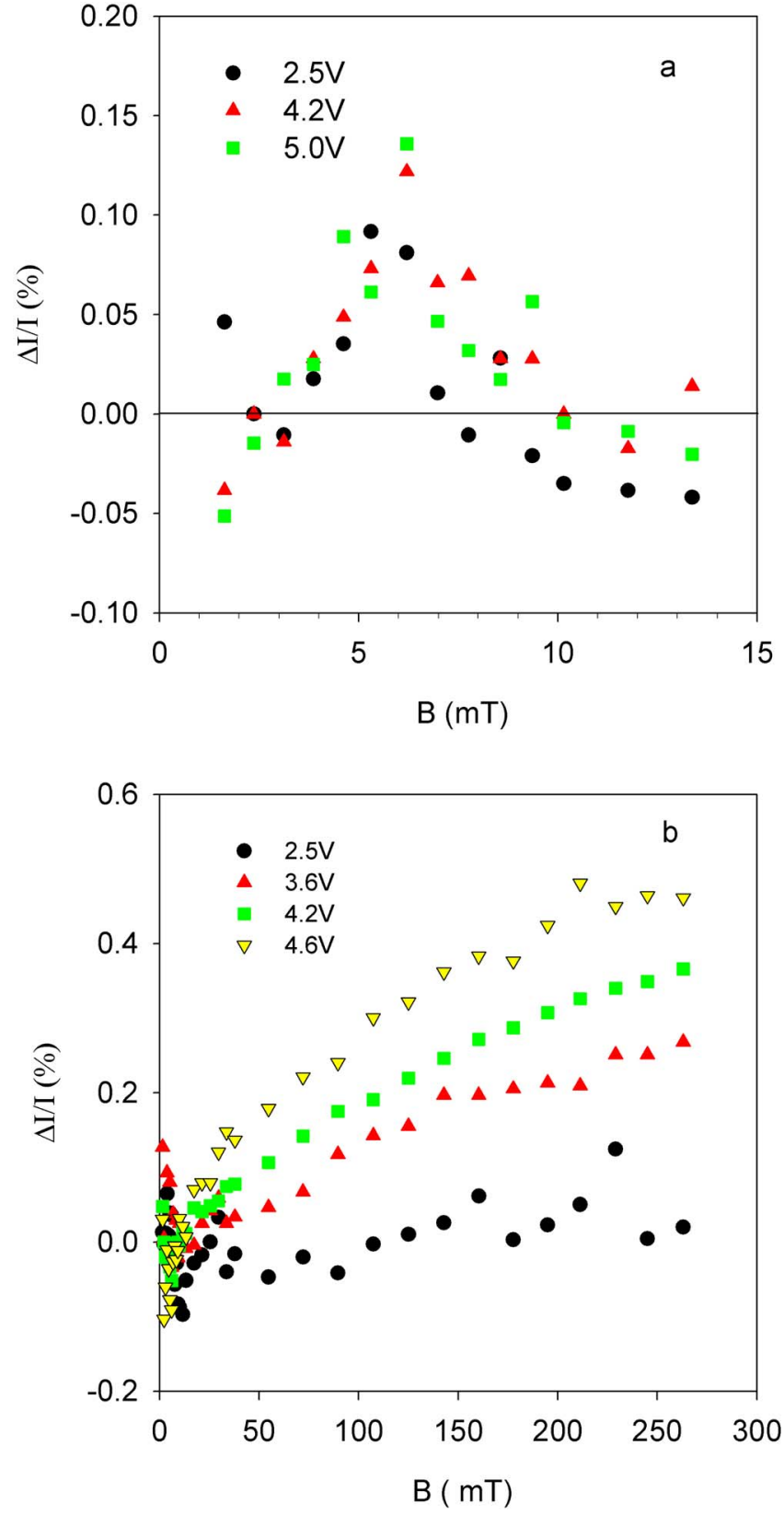

FIG. 4. (Color online) The percentage change in current at low magnetic field through $\mathrm{Ga}_{3}$ devices with thicknesses of (a) $200 \AA$ and (b) $500 \AA$.

For $\mathrm{Al}_{3}$ devices, it was seen that as the thickness of the $\mathrm{Al} q_{3}$ layer was reduced, the OMR just after turn-on was found to change from positive to negative. ${ }^{16}$ This was attributed to the role of triplet dissociation at the cathode as the insertion of an exciton blocking layer removed the effect. For $\mathrm{Al} q_{3}$, this dissociation related OMR was just visible for devices with a $200 \AA \mathrm{Al} q_{3}$ layer and dominated the OMR around turn-on for devices with $\mathrm{Al} q_{3}$ thicknesses of $150 \AA$ or less. For the $\mathrm{Ga}_{3}$ devices, similar behavior was observed, with a small dissociation related OMR, which was similar in shape to the positive efficiency change, observed at $200 \AA$. However, the effect was not significant until the $\mathrm{Ga} q_{3}$ device thickness was reduced to $100 \AA$ or less (Fig. 5). For $\operatorname{In} q_{3}$ devices, a dissociation process could be observed for the $150 \AA$ device but was very noisy and did not dominate the OMR even for devices with $\operatorname{In} q_{3}$ layers of $100 \AA$. This de- 

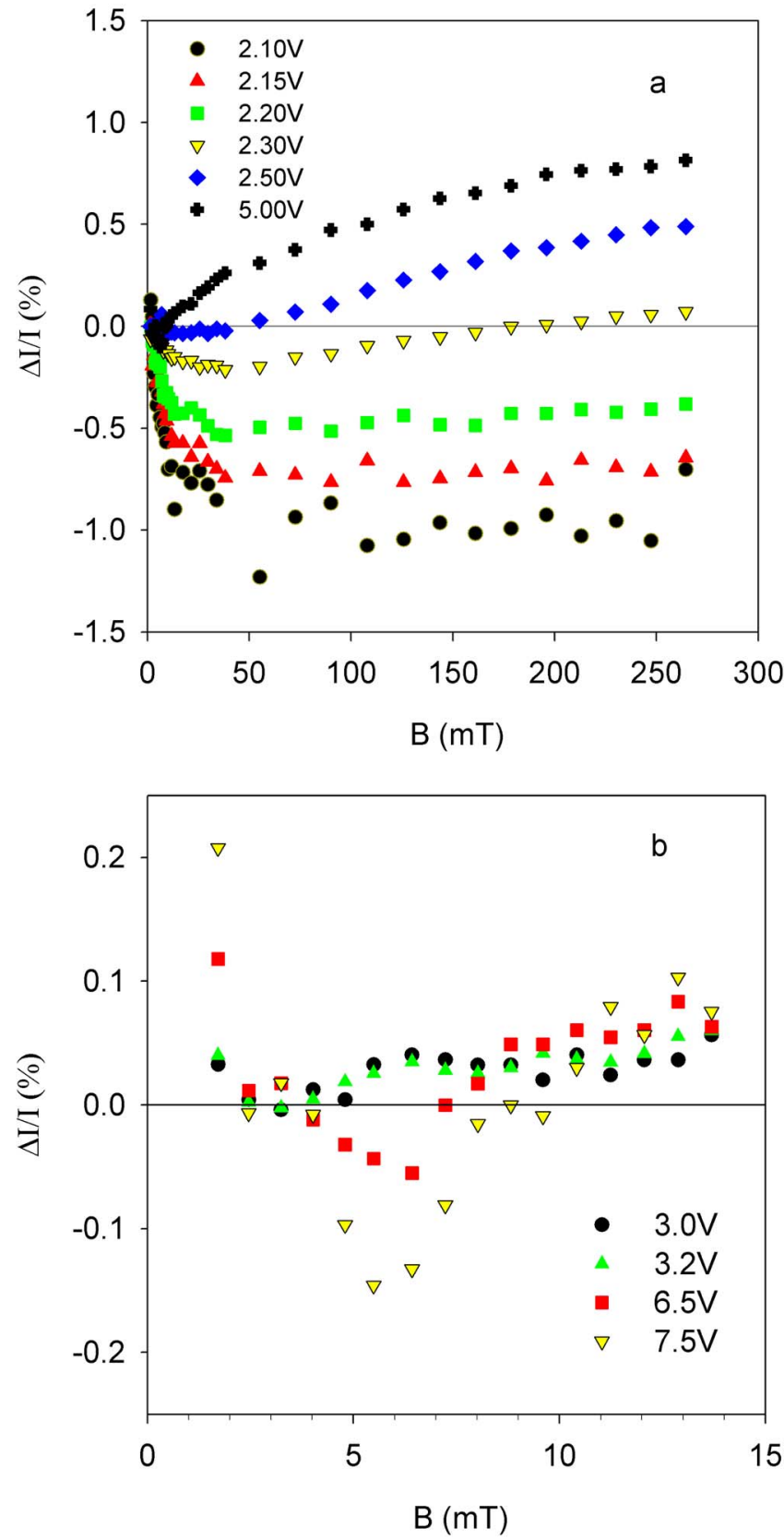

FIG. 5. (Color online) The percentage change in current through $10 \mathrm{~nm}$ devices of (a) $\mathrm{Ga}_{3}$ and (b) $\mathrm{In} q_{3}$ as a function of applied field for various drive voltages.

crease in the role of the dissociation of triplets in the devices with increasing mass of the group III ion could be due to the decrease in the triplet diffusion length. This could be brought about by the shorter lifetime due to the increase spin-orbit coupling as the mass of the group III ion is increased.

\section{CONCLUSIONS}

We have investigated the OMR of a series of OLED devices made from the group III quinolate complexes $\mathrm{Al}_{3}$, $\mathrm{Ga} q_{3}$, and $\mathrm{In} q_{3}$ as a function of the thickness of the quinolate layer. For all devices, the efficiency was found to increase with increasing magnetic field and could be fitted with a double "Lorentzian" function, which could possibly be related to the $B$ dependence of the hyperfine and spin-orbit contributions to the mixing between the singlet and triplet states.

For $\mathrm{Al}_{3}$ devices, the OMR was found to contain a term that was directly proportional to this change in efficiency along with another component that was very approximately linear with $B$. The component, which was proportional to the increase in efficiency, is consistent with the interaction of carriers with triplet states within the device. For $\mathrm{Ga} q_{3}$ and In $q_{3}$, there was no evidence of a component proportional to the efficiency increase and this could be due to there being a low energetic barrier to trapping of carriers at excitons in these materials at null field. For these materials, there was only a weak OMR but peaks in the OMR were visible at low magnetic fields. The mechanism behind these processes is not clear and will need further investigation.

The contribution of exciton dissociation at the cathode to the current through devices was also investigated as a function of materials and device thickness. Where this dissociation current was found to be quite significant for thin $\mathrm{Al} q_{3}$ devices, its effect was found to decrease as the mass of the central ion was increased. It was suggested that this could be due to a reduction in the exciton diffusion length with increasing ion mass because of the reduction in triplet lifetime due to the effect of the increased spin-orbit interaction.

${ }^{1}$ J. Kalinowski, M. Cocchi, D. Virgili, P. Di Marco, and V. Fattori, Chem. Phys. Lett. 380, 710 (2003).

${ }^{2}$ Ö. Mermer, G. Veeraraghavan, T. L. Francis, and M. Wohlgenannt, Solid State Commun. 134, 631 (2005).

${ }^{3}$ Ö. Mermer, M. Wohlgenannt, T. L. Francis, and G. Veeraraghavan, IEEE Trans. Magn. 41, 3682 (2005).

${ }^{4}$ Ö. Mermer, G. Veeraraghavan, T. L. Francis, Y. Sheng, D. T. Nguyen, M. Wohlgenannt, A. Köhler, M. K. Al-Suti, and M. S. Khan, Phys. Rev. B 72, 205202 (2005).

${ }^{5}$ Y. Sheng, D. T. Nguyen, G. Veeraraghavan, Ö. Mermer, M. Wohlgenannt, S. Qiu, and U. Scherf, Phys. Rev. B 74, 045213 (2006).

${ }^{6}$ T. D. Nguyen, Y. Sheng, J. Rybicki, G. Veeraraghavan, and M. Wohlgenannt, J. Mater. Chem. 17, 1995 (2007).

${ }^{7}$ V. N. Prigodin, J. D. Bergeson, D. M. Lincln, and A. J. Epstein, Synth. Met. 156, 757 (2006).

${ }^{8}$ P. A. Bobbert, T. D. Nguyen, F. W. van Oost, B. Koopmans, and M. Wohlgenannt, Phys. Rev. Lett. 99, 216801 (2007).

${ }^{9}$ P. Desai, P. Shakya, T. Kreouzis, W. P. Gillin, N. A. Morley, and M. R. J. Gibbs, Phys. Rev. B 75, 094423 (2007).

${ }^{10}$ R. P. Groff, R. E. Merrifield, A. Suna, and P. Avakian, Phys. Rev. Lett. 29, 429 (1972)

${ }^{11}$ R. P. Groff, A. Suna, P. Avakian, and R. E. Merrifield, Phys. Rev. B 9, 2655 (1974).

${ }^{12}$ R. E. Merrifield, J. Chem. Phys. 48, 4318 (1968).

${ }^{13}$ R. C. Johnson and R. E. Merrifield, Phys. Rev. B 1, 896 (1970).

${ }^{14}$ A. Suna, Phys. Rev. B 1, 1716 (1970).

${ }^{15}$ V. Ern and R. E. Merrifield, Phys. Rev. Lett. 21, 609 (1968).

${ }^{16}$ P. Desai, P. Shakya, T. Kreouzis, and W. P. Gillin, J. Appl. Phys. 102, 073710 (2007).

${ }^{17}$ P. Desai, P. Shakya, T. Kreouzis, and W. P. Gillin, Phys. Rev. B 76, 235202 (2007).

${ }^{18}$ P. E. Burrows, L. S. Sapochak, D. M. McCarty, S. R. Forrest, and M. E. Thompson, Appl. Phys. Lett., 64, 202718 (1994). 